\title{
MULTILEVEL LINC SYSTEM DESIGN FOR POWER EFFICIENCY ENHANCEMENT
}

\author{
Kai-Yuan Jheng, Yuan-Jyue Chen, and An-Yeu (Andy) Wu
}

\section{Graduate Institute of Electronics Engineering, National Taiwan University, Taipei 10617, Taiwan}

\begin{abstract}
Linear amplifier with nonlinear components (LINC) is a power amplifier (PA) linearization technique which offers both high PA efficiency and high linearity of wireless transmitters. But at the output stage, LINC uses a power combiner which results in low system efficiency. To solve this problem, we propose a multilevel out-phasing (MOP) scheme and a corresponding architecture, multilevel LINC (MLINC), to increase power combiner efficiency of wireless transmitters. Under WCDMA system linearity requirements, we demonstrate the 3-level MLINC as a design example which enhances power combiner efficiency from $44.5 \%$ to $75.5 \%$.
\end{abstract}

Index Terms - Linear amplifier with nonlinear components (LINC), out-phasing technique, power amplifier linearization.

\section{INTRODUCTION}

Linear amplifier with nonlinear components (LINC) [1] is a power amplifier (PA) linearization technique which can increase PA efficiency and linearity of wireless transmitters. Several LINC researches are proposed in recent years [2][5]. Fig. 1(a) shows the conventional LINC system. The signal component separator (SCS) decomposes a varyingenvelope signal $S(t)$ into two constant-envelope phasemodulated signals $S_{l}(t)$ and $S_{2}(t)$. Two modulators then upconvert these two baseband signals $S_{1}(t)$ and $S_{2}(t)$ to radio frequency $(\mathrm{RF})$. As $S_{1}(t)$ and $S_{2}(t)$ are constant-envelope signals, LINC utilizes two high-efficiency nonlinear PAs to amplify these signals. Finally, two amplified signals are combined with a power combiner. Thus, we can linearly amplify a varying-envelope signal with the conventional LINC.

To obtain the linearly amplified signal, LINC requires a power combiner at the output stage. When the lossy combiner such as Wilkinson combiner is used, this lowefficiency combiner leads to low system efficiency. Although we can use the lossless combiner such as Chireixoutphasing combiner for high efficiency, the lossless combiner degrades linearity significantly [6][7].

This work was supported in part by the MediaTek Inc., under NTU-MTK wireless research project.

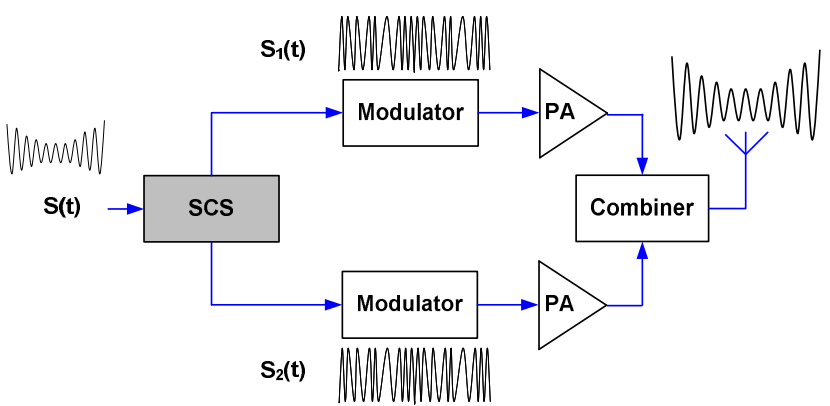

(a)

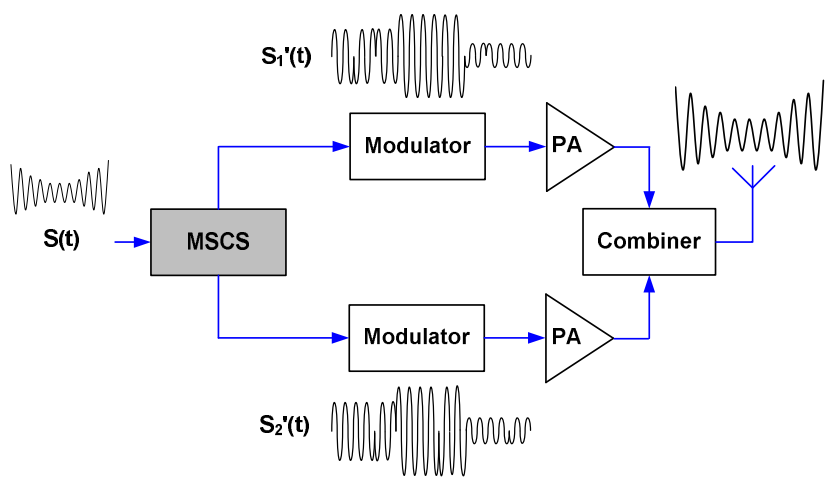

(b)

Fig. 1. The block diagram of (a) the conventional LINC system and (b) the proposed MLINC system.

In this paper, we design a multilevel LINC (MLINC) system to enhance the power combiner efficiency of LINC wireless transmitters. Fig. 1(b) shows the block diagram of the proposed MLINC system. Instead of the conventional LINC using a single-level SCS, MLINC utilizes a multilevel SCS (MSCS) to split $S(t)$ into two multilevel-envelope phase-modulated signals $S_{1}^{\prime}(t)$ and $S_{2}{ }^{\prime}(t)$ to achieve high Wilkinson combiner efficiency. In addition, inspired by the predistortion technique [8], we use a simplified predistorter to maintain acceptable linearity.

Our major contributions are outlined as follows.

1) We advance a multilevel out-phasing (MOP) scheme to enhance the combiner efficiency. (Section 2)

2) Based on the MOP scheme, we propose the MLINC architecture. (Section 3)

3) We demonstrate a system-level design example of the 3-level MLINC and compare the performances with conventional LINC under WCDMA system specifications. (Section 4) 


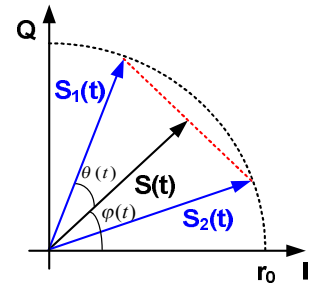

(a)

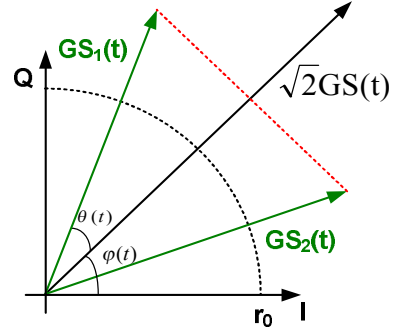

(b)

Fig. 2. Out-phasing concept: (a) before amplification and (b) after amplification.

\section{MULTILEVEL OUT-PHASING SCHEME}

To investigate the MOP scheme, the main idea of MLINC, we begin with an overview of the out-phasing technique. Next, we introduce the multilevel scaling (MS) technique which increases the combiner efficiency. We also derive each optimal scale factor set of MS to maximize combiner efficiency.

\subsection{Out-Phasing Technique}

The input of LINC systems is a amplitude and phasemodulated signal $S(t)$,

$$
S(t)=A(t) \cdot e^{j \varphi(t)}
$$

where $A(t)$ denotes the signal envelope and $\varphi(t)$ is signal phase. As the phasor diagram shown in Fig. 2(a), we can split the varying-envelope signal $S(t)$ into a set of constantenvelope signals, $S_{1}(t)$ and $S_{2}(t)$,

$$
\begin{aligned}
S(t) & =\frac{1}{2}\left[S_{1}(t)+S_{2}(t)\right] \\
& =\frac{1}{2} r_{0}\left[e^{j(\varphi(t)+\theta(t))}+e^{j(\varphi(t)-\theta(t))}\right]
\end{aligned}
$$

And the out-phasing angle $\theta(t)$ is expressed as

$$
\theta(t)=\cos ^{-1}\left(\frac{A(t)}{r_{0}}\right)
$$

Both $S_{1}(t)$ and $S_{2}(t)$ are on a circle whose radius is $r_{0}$. In conventional LINC transmitters, $r_{0}$ is a constant scale factor predefined by the system designers. Because the input range of inverse cosine function is $[-1,1]$, the selection of $r_{0}$ should satisfy (4),

$$
\mathrm{r}_{0} \geq \max (A(t))
$$

Fig. 2(b) illustrates the signals after amplification. The amplified signals are expressed as $G \cdot S_{1}(t)$ and $G \cdot S_{2}(t)$, where $G$ is the PA voltage gain. Then we can combine these

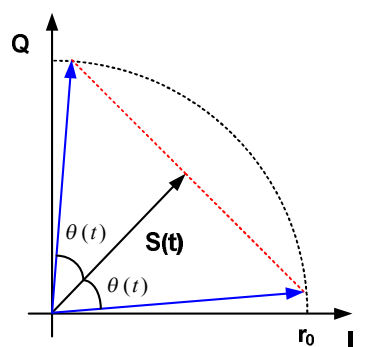

(a)

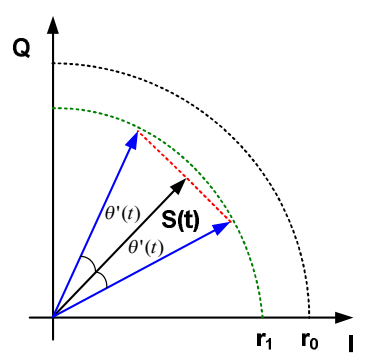

(b)
Fig. 3. Out-phasing angles in (a) single-level and (b) 2-level scaling technique.

two amplified signals with a Wilkinson combiner to obtain a signal $\sqrt{2} G \cdot S(t)$ which is a linear amplification of the input signal $S(t)$ [9]. Because of using this out-phasing technique, LINC can achieve linear amplification with two highefficiency nonlinear PAs.

As for the Wilkinson combiner efficiency $\eta(t)$ which is defined by (5)

$$
\begin{aligned}
\eta(t) & =\frac{|\sqrt{2} G \cdot S(t)|^{2}}{\left|G \cdot S_{1}(t)\right|^{2}+\left|G \cdot S_{2}(t)\right|^{2}} \\
& =\cos ^{2} \theta(t)
\end{aligned}
$$

we note that $\eta(t)$ is high when $\theta(t)$ is small. Then we substitute (3) into (5). This yields

$$
\eta(t)=\left(\frac{A(t)}{r_{0}}\right)^{2}
$$

From (4) and (6), we can conclude that for high $\eta(t)$, the designer should set $r_{0}$ equal to the maximum input signal envelope $\max (A(t))$.

\subsection{Multilevel Scaling Technique}

Instead of the traditional scaling technique using singlelevel scale factor $r_{0}$, MOP utilizes a multilevel scaling (MS) technique as a solution to shrink $\theta(t)$ to achieve high $\eta(t)$. We define the out-phasing angle $\theta^{\prime}(t)$ of MS as

$$
\theta^{\prime}(t)=\cos ^{-1}\left(\frac{A(t)}{R_{N}}\right)
$$

where

$$
\begin{aligned}
& R_{N}=r_{k}, \quad \text { for } \quad r_{k+1}<A(t) \leq r_{k} \quad k=0,1, \ldots, N-1, \\
& r_{N}=0, \quad r_{0}=\max (A(t))
\end{aligned}
$$

MS is a self-adjusting mechanism. According to (8), MS adapts multilevel scale factor $R_{N}$ to shrink out-phasing angle. The MS out-phasing angle $\theta^{\prime}(t)$ in Fig. 3(b) is much smaller than the conventional out-phasing angle $\theta(t)$ in Fig. 3(a), so MS can enhance the Wilkinson combiner efficiency $\eta(t)$. 


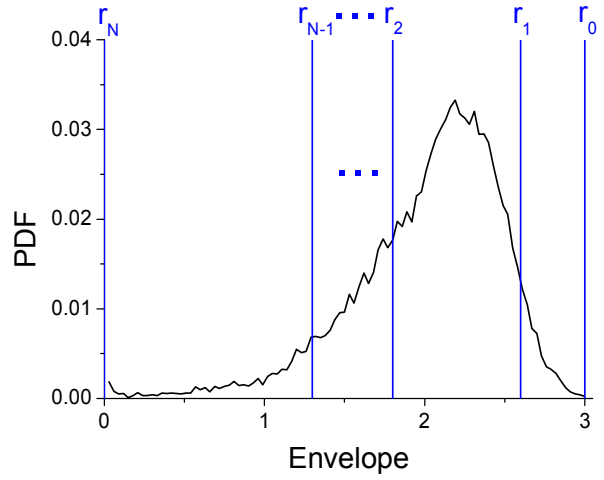

Fig. 4. Envelpoe distribution of WCDMA.

\subsubsection{Optimal Scale Factor Determination}

As we use MS to maximize the Wilkinson combiner efficiency, we have to determine the optimal value of each level $r_{k}$. We modify the Wilkinson combiner efficiency equation (7) as

$$
\eta(t)=\left(\frac{A(t)}{R_{N}}\right)^{2}
$$

Fig. 4 shows the WCDMA envelope distribution where $A(t)$ is a probability function. To get the expected value of $\eta(t)$, we divide the envelope $A(t)$ into several regions, which is illustrated in Fig. 4. Then, we sum up the expectation value of each region to derive $E(\eta(t))$,

$$
E(\eta(t))=\sum_{k=0}^{k=N-1} \int_{r_{k+1}}^{r_{k}} p(A(t)) \cdot\left(\frac{A(t)}{r_{k}}\right)^{2} d A(t)
$$

where $p(A(t))$ is the probability density function of $A(t), r_{k}$ is the value of $k$ th level scale factor, $N$ is the number of total scale factor level, and $\max (A(t))$ is the maximum input signal envelope. For the maximum Wilkinson combiner efficiency, we differentiate $E(\eta(t))$ as (11) to obtain the optimal set of $R_{N}$.

$$
\frac{\partial E(\eta(t))}{\partial R_{N}}=0
$$

With the optimal set of $R_{N}$, MS dynamically adapts $R_{N}$ close to and no smaller than the envelope $A(t)$ to enhance the Wilkinson combiner efficiency. MS can be easily implemented with a ROM table to save $R_{N}$, a comparator and a multiplexer to select the optimal value for $R_{N}$.

\subsection{Multilevel Predistortion Technique}

Since MS mechanism adjusts the multilevel envelope $R_{N}$, this introduces AM-AM distortion as Fig. 5(c). To compensate AM-AM distortion, we use a multilevel predistortion technique shown in Fig. 5(a). As illustrated in

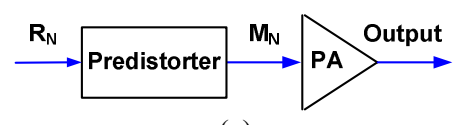

(a)

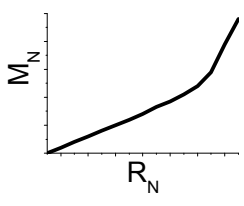

(b)

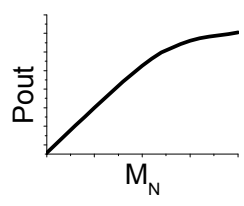

(c)

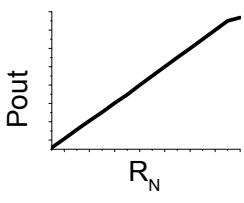

(d)
Fig. 5. (a) The concept of multilevel predistortion technique and the characteristic of (b) multilevel predistortion, (c) AM-AM model, and (d) output with predistortion.

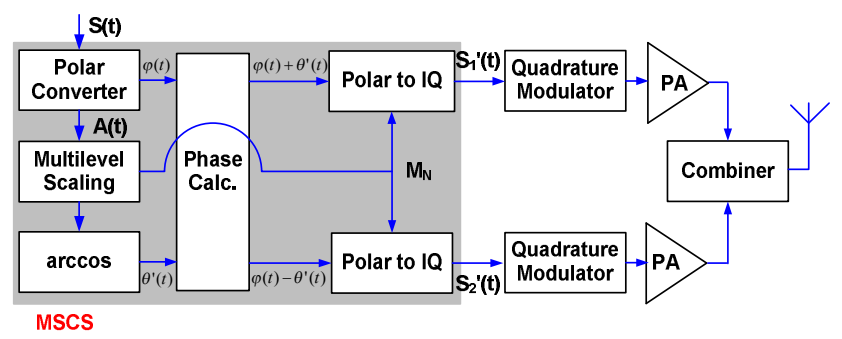

Fig. 6. The block diagram of MLINC architecture.

Fig. 5(b), we predistort the signal magnitude from $R_{N}$ to $M_{N}$ to obtain linear output as shown in Fig. 5(d).

\section{MLINC ARCHITECTURE}

The MLINC architecture is illustrated in Fig. 6. The shaded block is the MSCS which accomplishes the proposed MOP scheme. Firstly, baseband input signal $S(t)$ is converted to polar form by rectangular to polar converter. The envelope $A(t)$ is scaled by a multilevel scaling mechanism and outphasing angle $\theta^{\prime}(t)$ is obtained by an inverse cosine computation. The multilevel scaling mechanism also determines the predistorted envelope $M_{N}$. Then phase signals $\varphi(t)+\theta^{\prime}(t)$ and $\varphi(t)-\theta^{\prime}(t)$ and envelope signal $M_{N}$ are converted to rectangular form by polar to IQ converters. Two quadrature modulators are utilized to up-convert the baseband signals $S_{1}^{\prime}(t)$ and $S_{2}^{\prime}(t)$ to RF. Finally, the RF signals are amplified by two PAs and combined with a power combiner.

\section{SYSTEM SIMULATION}

The MLINC system simulations are based on Agilent Advanced Design System (ADS) and MathWorks MATLAB.

First, the system simulations set the $N$ from one to eight to verify the MS method. As shown in Fig. 7, while more than three scaling levels are used, most $\theta^{\prime}(t)$ range smaller than 38 degrees. In addition, using more scaling levels can make $\theta^{\prime}(t)$ smaller. Fig. 8 shows the Wilkinson combiner efficiency simulations of MLINC. MLINC enhances Wilkinson combiner efficiency from $44.5 \%$ to $88.9 \%$ with $N$ 


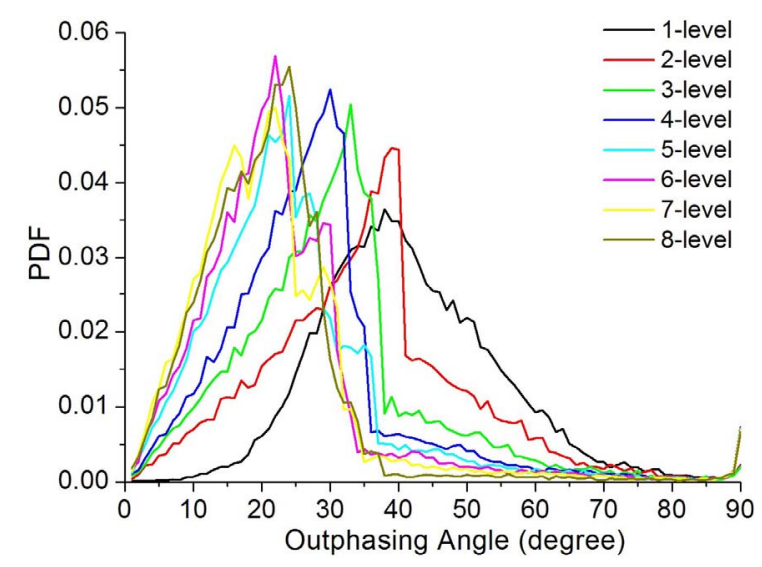

Fig. 7. Out-phasing angle distribution with different scaling levels.

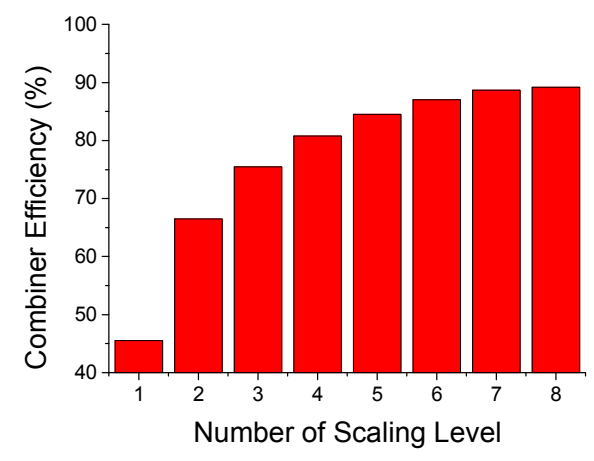

Fig. 8. Power efficiency of MLINC with different scaling levels.

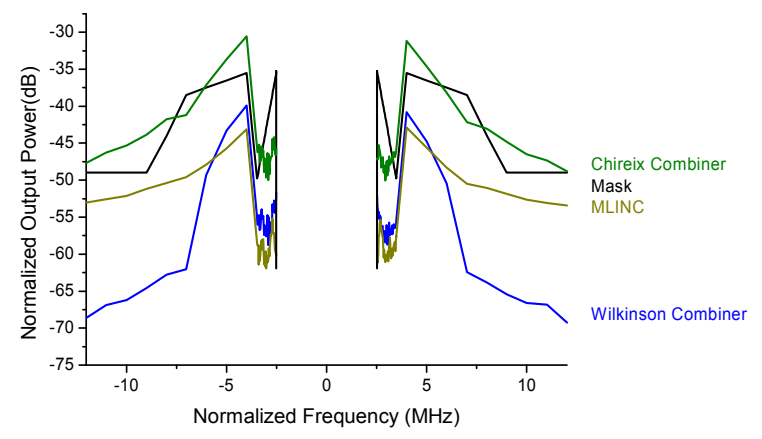

Fig. 9. Mixed-mode system simulation results of spectral emission.

from one to eight. In summary, using more scaling levels, MLINC can shrink $\theta^{\prime}(t)$ and enhance $\eta(t)$ further.

Next, we demonstrate a prototyping design using 3-level scaling in MLINC with a MOS-based RF PA model in ADS. We compare the proposed MLINC with conventional LINC using Wilkinson combiner or Chireix-outphasing combiner. The simulation results under WCDMA specifications are summarized in Table 1 and Fig. 9. Although Chireixoutphasing combiner improves the combiner efficiency to $78.8 \%$, it degrades the linearity significantly. Thus, using Chireix-outphasing combiner cannot pass the mask test of WCDMA specifications. In the case of our MLINC design
TABLE I

COMPARISONS OF POWER EFFICIENCY, EVM, AND MASK TEST

\begin{tabular}{cccc}
\hline \hline Architecture & $\begin{array}{c}\text { Power } \\
\text { Efficiency }\end{array}$ & EVM & $\begin{array}{c}\text { Mask } \\
\text { Test }\end{array}$ \\
\hline \hline $\begin{array}{c}\text { LINC with } \\
\text { Wilkinson Combiner }\end{array}$ & $44.5 \%$ & $1.2 \%$ & Pass \\
\hline $\begin{array}{c}\text { LINC with Chireix- } \\
\text { outphasing Combiner }\end{array}$ & $78.8 \%$ & $6 \%$ & Fail \\
\hline $\begin{array}{c}\text { MLINC } \\
\text { (3-level) }\end{array}$ & $75.5 \%$ & $3.2 \%$ & Pass \\
\hline \hline
\end{tabular}

example, it enhances the Wilkinson combiner efficiency from $44.5 \%$ to $75.5 \%$. Moreover, MLINC maintains high linearity that the error vector magnitude (EVM) is $3.2 \%$ which is less than $17.5 \%$ of WCDMA specifications and the spectral emission satisfies the spectral mask of WCDMA specifications.

\section{CONCLUSION}

A MLINC system is proposed to not only increase the Wilkinson combiner efficiency but also maintain high linearity of LINC transmitters. We also derive the optimal value of each scaling level to maximize the Wilkinson combiner power efficiency. Finally, we demonstrate the 3level MLINC as a design example which improves power combiner efficiency from $44.5 \%$ to $75.5 \%$ and maintains high linearity to fulfill WCDMA specifications.

\section{REFERENCES}

[1] D. C. Cox, "Linear amplification with nonlinear components," IEEE Trans. Commun., pp. 1942-1945, Dec. 1974.

[2] A. Birafane and R. H. Knoechel, "LINC digital component separator for single and multicarrier W-CDMA signals," IEEE Trans. Microw. Theory Tech., vol. 53, pp. 274-282, Jan. 2005.

[3] K.-Y. Jheng, Y.-C. Wang, A.-Y. Wu, and H.-W. Tsao, "DSP engine design for LINC wireless transmitter systems," in Proc. IEEE Int. Symp. Circuits Syst. (ISCAS), May 2006, pp. 2593-2596.

[4] F. Agnelli, et al., "Wireless multi-standard terminals: system analysis and design of a reconfigurable RF front-end," IEEE Circuits Syst. Mag., vol. 6, pp. 38-59, 2006.

[5] Y.-J. Chen, K.-Y. Jheng, A.-Y. Wu, H.-W. Tsao, and B. Tzeng, "Multilevel LINC system design for wireless transmitters," in Proc. IEEE Int. Symp. VLSI Design, Autom. Test (VLSI-DAT), Apr. 2007, pp. 208-211.

[6] A. Birafane and A. B. Kouki, "On the linearity and efficiency of outphasing microwave amplifiers," IEEE Trans. Microw. Theory Tech., vol. 52, pp. 1702-1708, Jul. 2004.

[7] A. Birafane and A. B. Kouki, "Phase-only predistortion for LINC amplifiers with Chireix-outphasing combiners," IEEE Trans. Microw. Theory Tech., vol. 53, pp. 2240-2250, Jun. 2005.

[8] N. Ceylan, J.-E. Mueller, and R. Weigel, "Optimization of EDGE terminal power amplifiers using memoryless digital predistortion," IEEE Trans. Microw. Theory Tech., vol. 53, pp. 515-522, Feb. 2005.

[9] D. M. Pozar, Microwave engineering, NJ: Wiley, 2005, pp. 318-323. 